\title{
Conditioning of odors in compound with taste is a function of factors other than potentiation
}

\author{
ROBIN L. LASHLEY and ROBERT A. ROSELLINI \\ State University of New York at Albany, Albany, New York
}

\begin{abstract}
Rats ingested odor-alone and/or odor-taste solutions and were subsequently made ill by lithium chloride injections. Following poisoning, aversions to the odor stimuli were assessed using a twobottle choice test. The results failed to provide clear evidence of odor-taste potentiation for all the stimuli we employed, regardless of the nature of the odor CSs administered and the use of both within- and between-group analyses. These findings suggest that previous reports of odortaste potentiation may be somewhat tenuous, and that odor saliency and order of conditioning may play more important roles than potentiation does in the development of odor aversion.
\end{abstract}

Previous data from our laboratory (Rosellini \& Lashley, 1986) suggest that enhanced aversion to an odor CS conditioned in compound with taste may be a function of odor saliency rather than potentiation, or perhaps an interaction between the two. In the present studies, therefore, we replicated the conditioning to odor-taste and odoralone solutions, but added groups which were conditioned to the two odors in isolation. This design allows us to separately assess the contributions of saliency and potentiation to the observed conditioning.

\section{EXPERIMENTS 1a, 1b, AND 1c}

In Experiment 1a, as in the previous studies, the odor (O) stimuli were $1.5 \% \mathrm{v} / \mathrm{v}$ solutions of Durkee brand banana extract (B) and Ann Page almond extract (A) in distilled water. Odor-taste (OT) compounds consisted of an odor in combination with a $0.06 \% \mathrm{w} / \mathrm{v}$ solution of sodium saccharin (S). To assess the possibility that banana and almond are atypical, two additional studies employed different odor combinations. A $1.5 \% \mathrm{v} / \mathrm{v}$ solution of Durkee brand strawberry extract (St) has previously been used by Durlach and Rescorla (1980). In Experiment 1b, subjects were conditioned to banana and strawberry solutions, and in Experiment 1c, to almond and strawberry solutions. Within each experiment, one group received one of the odors in compound with saccharin and the other odor in isolation; a second group received the reverse; and a third group received each odor in isolation.

\section{Method}

Subjects and Apparatus. One hundred twelve male Holtzman rats served as subjects. Age, housing, and apparatus were the same as those in the previous experiments (Rosellini \& Lashley, 1986).

This research was partially supported by NSF Grant No. BNS 7820678. We wish to thank Jeffery Carlson, Joseph P. DeCola, Mark Plonsky, Michael E. Abbot, and Anne Stilman for their assistance in this project. Requests for reprints should be sent to the first author at Kent State University, Tuscarawas Campus, New Philadelphia, $\mathrm{OH}$ 44663.
Procedure. In Experiment 1a, Group BS/A $(N=8)$ was conditioned to banana-saccharin and to almond; Group AS/B $(N=8)$ to almondsaccharin and to banana; and Group B/A $(N=16)$ to the banana and almond odors in isolation. In Experiment $1 \mathrm{~b}$, Group BS/St $(N=8)$ was conditioned to banana-saccharin and to strawberry; Group StS/B $(N=8)$ to strawberry-saccharin and to banana; and Group $\mathrm{B} / \mathrm{St}(N=16)$ to the isolated banana and strawberry odors. In Experiment 1c, Group AS/St $(N=8)$ was conditioned to almond-saccharin and to strawberry; Group StS/A $(N=8)$ to strawberry-saccharin and to almond; and Group $\mathrm{A} / \mathrm{St}$ $(N=32)$ to isolated almond and strawberry odors. All procedures were identical to those of the earlier studies (Rosellini \& Lashley, 1986).

\section{Results}

Experiment 1a. The top panel of Figure 1 shows the mean intake of the B and A elements on the 2 test days as a function of group and poisoning order (OT or O conditioned first). The results were consistent with previous results: animals conditioned either to BS/A or to A and $B$ in isolation consumed less banana than almond, whereas those conditioned to AS/B showed equal consumption of $A$ and $B$. This was the case regardless of poisoning order. Analysis of variance as a function of group (BS/A, AS/B, or $\mathrm{B} / \mathrm{A}$ ), poisoning order (OT or $\mathrm{O}$ conditioned first), and test odor (B or A) showed a significant effect of test odor $[F(1,26)=16.11, p<.001]$, indicating stronger rejection of $B$ than $A$, and a significant group $\times$ test odor interaction $[F(2,26)=3.51, p=.045]$. Simple effects analyses indicated that although Groups B/A and BS/A consumed less $\mathrm{B}$ than $\mathrm{A}[F(1,14)=10.55, p=.006$; and $F(1,6)=19.15, p=.005$, respectively], Group AS/B showed no differences $(p>.20)$.

Comparison with the odors-only group allows us to differentiate between saliency and potentiation. With these animals, higher rejection of B must be attributed to its greater saliency. If potentiation also exerts an effect, then subjects in the BS/A group should exhibit a still higher rejection of B. Similarly, the aversion to A should be greater for the AS/B group than for the B/A group. However, comparison of intake of $B$ between Groups B/A and $B S / A$, as well as that of $A$ between Groups $B / A$ and AS/B, failed to show any significant differences (all $p s>.20)$. These results confirm that $A$ and $B$ differ in 

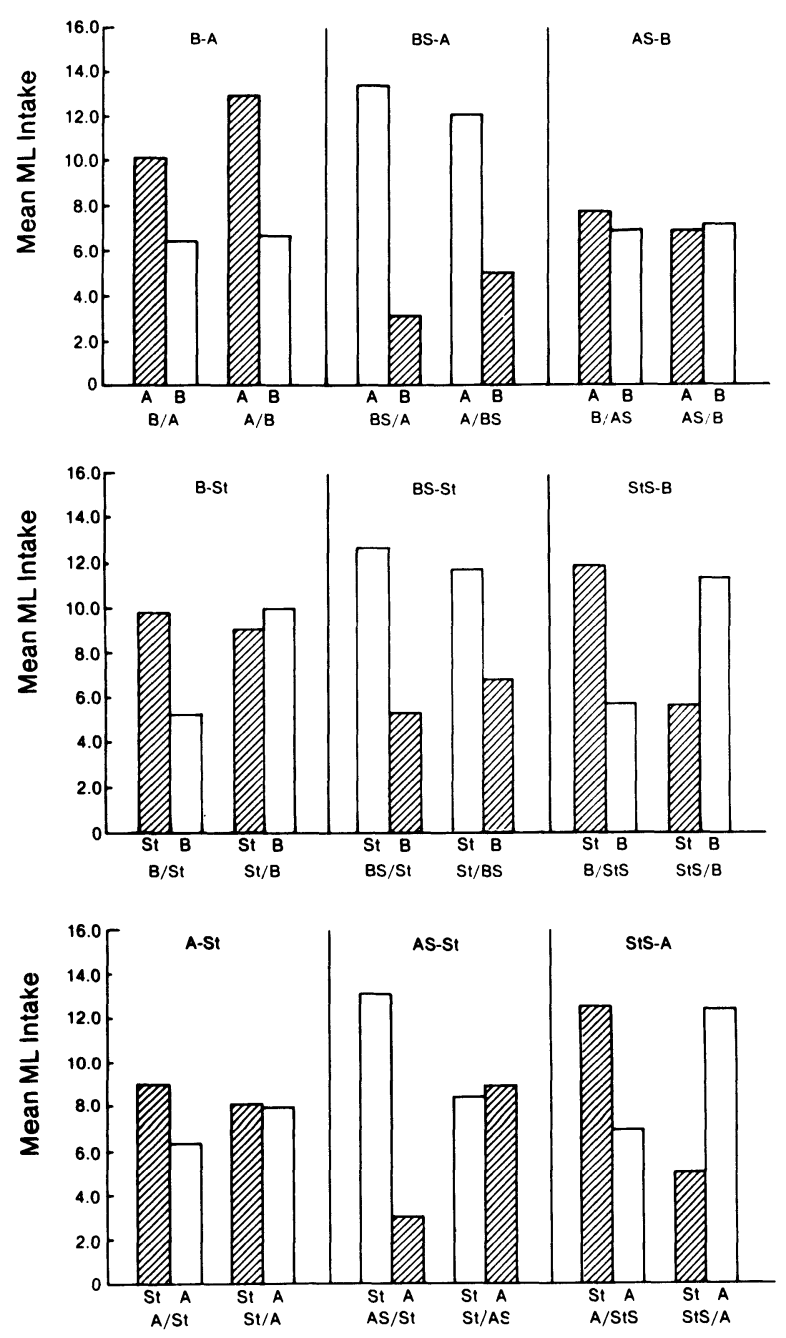

Figure 1. Upper panel: mean milliliter intake of banana- and almond-scented water for Groups B/A, BS/A, and AS/B as a function of conditioning order. Center panel: Mean milliliter intake of banana- and strawberry-scented water for Groups B/St, BS/St, and StS/B as a function of conditioning order. Lower panel: Mean milliliter intake of almond- and strawberry-scented water for Groups $\mathrm{A} / \mathrm{St}, \mathrm{AS} / \mathrm{St}$, and StS/A as a function of conditioning order. The hatched bars in the middle and right sections of each panel represent intake of the odor conditioned in compound with saccharin.

saliency, as evidenced by the stronger rejection of $B$ when both are conditioned in isolation. However, no real evidence of potentiation has been observed.

Experiment 1b. The center panel of Figure 1 shows mean intake of the banana and strawberry solutions on the 2 test days as a function of group and poisoning order. As was the case with almond, Group BS/St tended to consume less B, regardless of poisoning order. However, Group StS/B ingested less of the odor which had been conditioned first, regardless of whether it had been alone or in compound with taste. Animals conditioned to both odors in isolation (Group B/St) showed lower intake of $\mathrm{B}$ only if it had been conditioned first. Analysis of variance as a function of group $(\mathrm{BS} / \mathrm{St}, \mathrm{StS} / \mathrm{B}$, or $\mathrm{B} / \mathrm{St})$, poisoning order (OT or $\mathrm{O}$ conditioned first), and test odor (B or St) showed a significant effect of test odor $[F(1,26)$ $=4.54, p=.043$ ], indicating overall stronger rejection of $B$ than St, and a significant test odor $\times$ poisoning order interaction $[F(1,26)=6.48, p=.017]$. Simple effects analyses were conducted for each group separately. Group B/St showed no differential intake of the odors as a function of either test odor or poisoning order (all $p s>.10)$. Group BS/St showed a marginally higher rejection of B than of St $[F(1,6)=3.98, p=.093]$ and no effect of poisoning order $(p s>.20)$. Group StS/B showed only a significant test odor $\times$ poisoning order interaction $[F(1,6)=7.13, p=.037]$, indicating that the odor conditioned first was rejected more often, regardless of its taste history. Separate comparisons for each poisoning order of intake of B in Groups B/St and BS/St, and of St in Groups B/St and StS/B, failed to show any significant differences (all $p s>.20$ ).

Experiment 1c. The lower panel of Figure 1 shows the mean intakes of almond and strawberry on the 2 test days as a function of group and poisoning order. Animals conditioned to the isolated odors (Group $\mathrm{A} / \mathrm{St}$ ) consumed equivalent amounts of the two stimuli. Group AS/St showed stronger rejection of A only if it had been conditioned first, whereas Group StS/A rejected the odor which had been conditioned first regardless of its taste history. Analysis of variance as a function of group (AS/St, StS/A, or $\mathrm{A} / \mathrm{St}$ ), poisoning order (OT or $\mathrm{O}$ conditioned first), and test odor (A or St) showed a significant effect of test odor $[F(1,42)=4.09, p=.05]$, a significant poisoning order $\times$ test odor interaction $[F(1,42)=19.94$, $p<.001]$, and a significant group $\times$ poisoning order $\times$ test odor interaction $[F(2,42)=4.35, p=.019]$. Simple effects analyses conducted for each group separately showed that Group A/St had equivalent intakes of the two odors (all $p s>.15$ ). Group AS/St showed a significant poisoning order $\times$ test odor interaction $[F(1,6)=9.40$, $p=.022$ ], indicating that A was only rejected more when the AS compound was conditioned first. Group StS/A showed stronger rejection of the odor which had been conditioned first, regardless of whether this had been in compound or isolation, as indicated by a significant poisoning order $\times$ test odor interaction $[F(1,6)=14.03$, $p=.01]$.

Between-group comparisons for Groups $\mathrm{A} / \mathrm{St}$ and $\mathrm{AS} / \mathrm{St}$, conducted for each poisoning order separately, showed equivalent intakes of $\mathrm{A}(p s>.20)$. Groups $\mathrm{A} / \mathrm{St}$ and StS/A did not show any differential intake of St when it had been conditioned first $(p>.20)$, but if conditioned second, Group StS/A showed a marginally higher intake $[F(1,42)=3.17, .05<p<.10]$. This result is in the direction of overshadowing rather than potentiation.

\section{Discussion}

These studies show little evidence of potentiation of conditioning to odor by taste, at least with the odors employed here. This is the case whether potentiation is assessed using the within-group procedure of Durlach and Rescorla (1980), or the between-group comparisons as in Rusiniak, Hankins, Garcia, and Brett (1979). 
The outcome of Experiment 1a supports the earlier suggestion that banana and almond odors-at least at the $1.5 \% \mathrm{v} / \mathrm{v}$ concentration-differ in saliency, as evidenced by the stronger rejection of the former when both are conditioned in isolation. Experiment $1 \mathrm{~b}$ indicates that banana is also more salient than strawberry, and Experiment $1 \mathrm{c}$ indicates that almond and strawberry are approximately equivalent. Poisoning order also appears to be a more important determinant than whether the odor was conditioned in compound or in isolation.

Because these studies strongly suggest that odor saliency plays an important role, we conducted a final series of experiments to determine whether potentiation is demonstrated when this factor is equated.

\section{EXPERIMENTS 2a, 2b, AND 2c}

These studies replicated Experiments 1a, 1b, and 1c, with the difference that odor intensities were adjusted so that conditioning of isolated odors would result in equivalent rejection of each.

\section{Method}

Subjects and Apparatus. The subjects were 117 male Holtzman rats. Age, apparatus, and housing were the same as those described earlier.

Procedure. Experiments $2 \mathrm{a}, 2 \mathrm{~b}$, and $2 \mathrm{c}$ were identical to Experiments $1 \mathrm{a}, 1 \mathrm{~b}$, and 1c, respectively, except for the odor intensities employed. The concentrations used were a $.25 \% \mathrm{v} / \mathrm{v}$ solution of banana, a $.5 \% \mathrm{v} / \mathrm{v}$ solution of almond, and a $.5 \% \mathrm{v} / \mathrm{v}$ solution of strawberry, as pilot work had demonstrated that these intensities result in approximately equivalent aversions when conditioned in isolation. The compounds were combinations of these odors with a $.06 \% \mathrm{w} / \mathrm{v}$ solution of saccharin.

In Experiment 2a, Groups B/A and AS/B each contained 12 animals and Group BS/A contained 14; in Experiment 2b, Groups BS/St and StS/B each contained 14 animals and Group B/St contained 16; and in Experiment 2c, Groups AS/St and StS/A each contained 11 animals and Group A/St contained 13 .

\section{Results}

Experiment 2a. The top panel of Figure 2 shows the mean intake of banana and almond for the three groups as a function of poisoning order. As can be seen, no differential intake of the odors was observed. Analysis of variance as a function of group, poisoning order, and test odor, as well as subsequent simple effects analyses, failed to show any significant effects or interactions (all ps $>$.10).

Experiment 2b. The center panel of Figure 2 shows the mean intakes of banana and strawberry. Analysis of variance as a function of group (BS/St, StS/B, or B/St), poisoning order (OT or $\mathrm{O}$ conditioned first), and test odor (B or St) showed a significant poisoning order $\times$ test odor interaction $[F(1,38)=9.75, p=.003]$. Simple effects analyses conducted for each group separately showed only a significant poisoning order $\times$ test odor interaction for Group StS/B $[F(1,12)=9.17, p=.01]$, indicating a stronger rejection of $B$ than St when the former had been conditioned first, and no differential intake when it had been conditioned second. No between-group comparisons were significant.

Experiment 2c. The lower panel of Figure 2 shows the mean intakes of strawberry and almond. Analysis of variance as a function of group ( $\mathrm{AS} / \mathrm{St}, \mathrm{StS} / \mathrm{A}$, or $\mathrm{A} / \mathrm{St}$ ), poisoning order (OT or $\mathrm{O}$ conditioned first), and test odor (A or St) showed a significant group $\times$ test odor interaction $[F(2,29)=3.91, p=.031]$, a significant poisoning
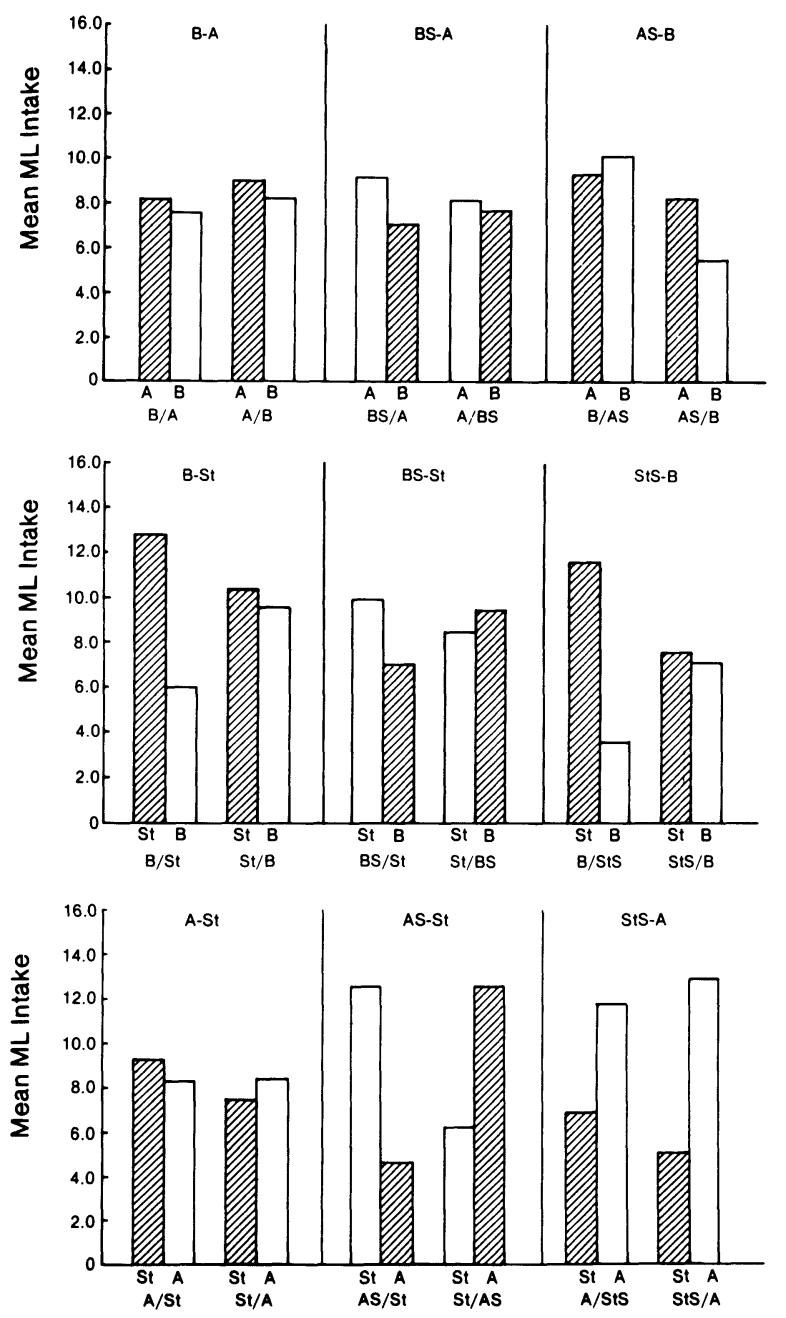

Figure 2. Upper panel: Mean milliliter intake of banana- and almond-scented water for Groups B/A, BS/A, and AS/B as a function of conditioning order. Center panel: Mean milliliter intake of banana- and strawberry-scented water for Groups B/St, BS/St, and StS/B as a function of conditioning order. Lower panel: Mean milliliter intake of almond- and strawberry-scented water for Groups $\mathrm{A} / \mathrm{St}, \mathrm{AS} / \mathrm{St}$, and StS/A as a function of conditioning order. The hatched bars in the middle and right sections of each panel represent intake of the odor conditioned in compound with saccharin.

order $\times$ test odor interaction $[F(1,29)=7.75, p=.009]$, and a marginally significant three-way interaction $[F(2,29)$ $=3.07, p=.062]$. Simple effects analyses conducted for each group separately showed Group A/St not to differ in intake of the two odors (all $p s>.20$ ). Group AS/St showed greater rejection of the odor which had been conditioned first regardless of its taste history, as demonstrated by a significant poisoning order $\times$ test odor interaction $[F(1,9)=8.68, p=.016]$. Group StS/A showed significantly greater rejection of St which had been conditioned in compound than A which had been conditioned in isolation, as indicated by a significant effect of test odor $[F(1,9)=8.02, p=.02]$, regardless of poisoning order. Subsequent between-group comparisons failed to reveal any differential intakes (all $p s>.20$ ). 


\section{Discussion}

As expected on the basis of pilot work, the intensities employed here resulted in equivalent amounts of conditioning to the isolated odors, at least in Experiments $2 \mathrm{a}$ and $2 \mathrm{c}$. Even so, potentiation was observed only in Group StS/A, which consumed less St conditioned in compound than A conditioned in isolation, regardless of which had been poisoned first. Unfortunately, even this one demonstration is ambiguous, since betweengroup comparisons of the intake of St by Groups A/St and StS/A indicated equivalent consumption by animals in these groups. Furthermore, potentiation was not observed on either a within-group or a betweengroup basis in Group AS/St. In this group, poisoning order was the critical determinant of associative strength. This failure to observe generality of potentiation across odors that were demonstrated to be equivalent in saliency is clearly problematic.

\section{GENERAL DISCUSSION}

Overall, these studies achieved little success in demonstrating the potentiation of conditioning to odor by taste. This is the case whether the target odor was contrasted with different odors, whether the contrasting odors were equated for stimulus saliency, or whether the data were assessed on a within-group or a between-group basis. In only one condition, Group StS/A of Experiment 2c, was the effect observed, at least on a within-group basis. Yet even this demonstration is questionable, since between-group comparisons failed to yield the same conclusion.

Whereas the stronger rejection of banana by animals in Group BS/A in Experiment 1a, which replicates our previous results (Rosellini \& Lashley, 1986), might also be viewed as an instance of potentiation, the results of Experiment 2a, in which the two odors were equated for saliency, suggest that the differential rejection of banana in the earlier studies is due to differential saliency. When the two odors are experienced as equivalent in intensity, then poisoning order appears to determine the extent of conditioning (Experiments $1 \mathrm{c}, 2 \mathrm{~b}$, and $2 \mathrm{c}$ ). This result is consistent with other reports of diminished effectiveness of the toxicosis US with repeated exposure (e.g., Riley, Jacobs, \& LoLordo, 1976).

Although these studies highlight the importance of saliency and poisoning order in the potentiation paradigm, they do not uncover the basic underlying factors. The apparent fragility of the phenomenon raises the question of whether it does, in fact, pose a substantial threat to established theories of Pavlovian conditioning (Frey \& Sears, 1978; Mackintosh, 1975; Rescorla \& Wagner, 1972). The critical combination of variables necessary to produce potentiation of conditioning to odor by taste remains to be discovered.

\section{REFERENCES}

Durlach, P. J., \& Rescorla, R. A. (1980). Potentiation rather than overshadowing in flavor-aversion learning: An analysis in terms of within-compound associations. Journal of Experimental Psychology: Animal Behavior Processes, 6, 323-332.

FrEY, P. W., \& SEARS, R. J. (1978). Model of conditioning incorporating the Rescorla-Wagner associative axiom, a dynamic attention rule, and a catastrophe rule. Psychological Review, 85, 321-340.

Mackintosh, N. J. (1975). A theory of attention: Variations in the associability of stimuli with reinforcers. Psychological Review, 82, 276-298.

Rescorla, R. A., \& Wagner, A. R. (1972). A theory of Pavlovian conditioning: Variations in the effectiveness of reinforcement and nonreinforcement. In A. H. Black and W. K. Prokasy (Eds.), Classical conditioning II: Current research and theories. New York: AppletonCentury-Crofts.

Riley, A. L., JACOBS, W. J., \& LoLordo, V. M. (1976). Drug exposure and the acquisition and retention of a conditioned taste aversion. Journal of Comparative \& Physiological Psychology, 90, 799-807.

Rosellini, R. A., \& LASHLey, R. L. (1986). Conditioning of odors in compound with taste: A failure to observe potentiation. Bulletin of the Psychonomic Society, 24, 55-58.

Rusiniak, K. W., Hankins, W. G., Garcia, J., \& Brett, L. P. (1979). Flavor-illness aversions: Potentiation of odor by taste in rats. Behavioral \& Neural Biology, 25, 1-17.

(Manuscript received for publication September 28, 1985.) 